\title{
Synthesis, Characterization, and Immunological Properties of LPS-Based Vaccines Composed of 0-Polysaccharides Conjugated with Recombinant Exoprotein A from Pseudomonas aeruginosa
}

\author{
Nareman F. Abu-Baker, Hussein Masoud* \\ Department of Biological Sciences, Faculty of Science, University of Jordan, Amman, Jordan \\ Email: *hmasoud@ju.edu.jo
}

Received 2 March 2016; accepted 17 April 2016; published 20 April 2016

Copyright (C) 2016 by authors and Scientific Research Publishing Inc.

This work is licensed under the Creative Commons Attribution International License (CC BY). http://creativecommons.org/licenses/by/4.0/

(c) (i) Open Access

\section{Abstract}

Pseudomonas aeruginosa remains one of the major pathogens affecting immunocompromised patients. LPS-based monovalent (MV) and polyvalent (PV) conjugate vaccines were prepared from the most prevalent strains of $P$. aeruginosa International Antigenic Typing Scheme (IATS) 6, 10, 11 and 20 to evaluate their immunogenicity and protective capacities from infection by the pathogens. Conjugation of the 0-polysaccharide (O-PS) antigens of $P$. aeruginosa strains to the common immunogenic recombinant Exotoxin A (rEPA) supports the multi-antigenic approach for the development of a vaccine that provides cross protection against various strains of the pathogen. The O-PSs were indirectly conjugated through adipic acid dihydrazide (ADH) to rEPA by carbodiimidemediated condensation reaction. Mice were immunized with the conjugates emulsified with monophosphoryl lipid A (MPL) or Freund's adjuvant compared with conjugates without adjuvant, unconjugated mixture of rEPA and O-PS emulsified with MPL, and sterile saline. The MV and PV vaccines emulsified with MPL adjuvant elicited the highest anti-0-PS IgM and IgG antibodies. Immunization of mice with MV vaccines derived from IATS 10, 11, and 20, emulsified with MPL adjuvant provided a high level of protection against the homologous bacterial strain. Similarly, high protection was obtained when mice were immunized using PV and challenged separately with bacterial strains 10, 11, and 20, but lower protection against the IATS 6 strain. Also, high cross protection of MV vaccine derived from 0-PS of IATS 10 and 20 was obtained against $P$. aeruginosa IATS 11 strain. The in vivo protection correlated with the level of anti-0-PS IgG in the mice serum.

${ }^{*}$ Corresponding author.

How to cite this paper: Abu-Baker, N.F. and Masoud, H. (2016) Synthesis, Characterization, and Immunological Properties of LPS-Based Vaccines Composed of O-Polysaccharides Conjugated with Recombinant Exoprotein A from Pseudomonas aeruginosa. Advances in Microbiology, 6, 332-342. http://dx.doi.org/10.4236/aim.2016.64032 


\section{Keywords}

\section{Pseudomonas aeruginosa, Lipopolysaccharide, Recombinant Exoprotein A, Conjugate Vaccine, Immunization}

\section{Introduction}

Pseudomonas aeruginosa is a major cause of morbidity and mortality for immunocompromised patients, especially in individuals with cystic fibrosis, severe burns, or cancer [1] [2]. In these patients, P. aeruginosa is one of the most dangerous nosocomial pathogens, due to its production of several virulence factors such as exotoxin A, exoenzyme S, elastase, alginate and lipopolysaccharide (LPS) [3] [4]; emergence of multidrug resistant strains [5]; and absence of an efficient protective vaccine [2].

Several candidate vaccines for $P$. aeruginosa have been evaluated, including capsular polysaccharide, low doses of LPS, purified surface proteins and whole live-attenuated cells, but none were successful in protecting from infection by this pathogen [2] [6]. However, some protections were observed using alginate-base conjugates in experimental animals [7] [8].

LPS is an important immunoreactive surface antigen of P. aeruginosa [6] [9], and is composed of a hydrophilic O-polysaccharide (O-PS) region that is linked to a hydrophobic lipid A via a core oligosaccharide. Twenty major serotypes of $P$. aeruginosa have been identified on the basis of their antigenic O-PS cross reactivity and chemical structure 0 [10]. The serum antibodies against the surface O-PS antigen confer protective immunity against the pathogen. Since intact LPS of Gram-negative bacteria is highly toxic, and isolated O-PS molecules are poor immunogens, especially in infants, thus many investigators have attempted to conjugate O-PS of pathogenic bacteria to immunogenic proteins to enhance the immunogenicity of O-PS and confer protection against the pathogen [11] [12].

Several immunogenic proteins, including tetanus toxoid (TT), diphtheria toxoid, $\mathrm{CRM}_{197}$, bovine serum albumin (BSA) and exotoxin A have been used as carrier proteins [12]-[16]. Exotoxin A is highly toxic and immunogenic and is produced by most clinical strains of $P$. aeruginosa [17]. Berna Biotech developed an octavalent conjugate vaccine $\left(\right.$ Aerugen ${ }^{\circledR}$ ) prepared by conjugation of the O-PSs from $P$. aeruginosa strains to exotoxin A. The vaccine was failed to produce sufficient efficacy in Phase 3 clinical trials to protect from $P$. aeruginosa infection in cystic fibrosis patients, thus further development of the vaccine was terminated [2].

Recombinant exotoxin A of $P$. aeruginosa (rEPA) is nontoxic and retains its immunogenic property, thus making rEPA a good candidate to be used as a vaccine and as a carrier protein for preparation of conjugate vaccines [18]-[21]. Using rEPA as a carrier protein supports a multi-antigenic approach for the development of a conjugate vaccine that elicits antibodies against both O-PS and rEPA of pathogenic P. aeruginosa, to provide broad spectrum protection from $P$. aeruginosa infection.

In this study, the O-PS from the most prevalent strains of $P$. aeruginosa, International Antigenic Typing Scheme (IATS) strains 6, 10,11, and 20 [3] [10], was used to create and test conjugation vaccines. rEPA was used as a carrier protein because of its safety, immunogenicity, and possibility for serological cross-protection between various strains. The immunogenicity of the monovalent (MV) and tetravalent conjugate vaccines emulsified with various adjuvants was evaluated in mice. In addition, the in vivo protection of mice was evaluated.

\section{Materials and Methods}

\subsection{Bacterial Growth and Preparation of LPS and 0-PS}

P. aeruginosa IATS strains 6, 10, 11, and 20 were kindly provided by Professor Joseph Lam (Department of Molecular and Cellular Biology, University of Guelph, Guelph, Canada). Cultivation and LPS preparation were performed as previously described [12] [22]. The purity of LPS was checked by testing for the presence of DNA [23] and protein [24]. The O-PS was obtained and purified as previously described [12].

\subsection{Carrier Protein}

Nontoxic rEPA was kindly provided by Dr. Ali Fattom (NABI Biopharmaceutical Company, Bethesda, USA). 
A preliminary test was performed to estimate the toxicity of the rEPA carrier protein. Samples of rEPA (50, 75, 200 , and $400 \mu \mathrm{g}$ in $0.2 \mathrm{ml}$ sterile PBS) were injected intraperitoneally (i.p.) into five BALB/c female mice weighing $17-20 \mathrm{~g}$. The animals were monitored for any sign of illness or weight loss over 7 days.

\subsection{Preparation of Conjugates}

The O-PSs of $P$. aeruginosa IATS strains 6, 10, 11, and 20 were coupled to the rEPA protein through adipic acid dihydrazide (ADH; Sigma, St. Louis, MO, USA). The rEPA was first activated with ADH by a carbodiimidemediated condensation reaction as previously described [13].

For the MV conjugate vaccine, the O-PSs of $P$. aeruginosa IATS strains were coupled through the free carboxyl group of 2-keto-3-deoxyoctulosonic acid (KDO) to the free amino group of the ADH-rEPA complex by a carbodiimide-mediated condensation reaction [25]. For the polyvalent (PV) conjugate vaccine, a mixture of O-PSs (7.5 mg from each strain) was conjugated as described above. The O-PS-ADH-rEPA conjugate was purified as described earlier [12], and concentrated by ultrafiltration, aliquoted, and stored at $-70^{\circ} \mathrm{C}$. The molar ratios of the protein to the saccharide moiety were calculated based on the molecular weight of rEPA (66,000 Da) and the molecular weight of the saccharide moiety, which is the total molecular weight of the complete core region [26] and one repeating unit of the O-PS for the corresponding strain of P. aeruginosa [10], based on that the most abundant LPS species contains one repeating unit, as shown by gel electrophoresis [27]. The average molecular weight of the saccharide moieties was used to calculate the molecular weight of the saccharide moiety in the PV.

\subsection{Mouse Immunization}

Stock conjugate solutions of O-PS were prepared at a concentration equivalent to $25 \mu \mathrm{g} / \mathrm{ml}$ in PBS. The conjugate solutions were sterilized by filtration (membrane pore size: $0.45 \mu \mathrm{m}$ ), emulsified in a 1:1 ratio with an adjuvant (Freund's or MPL), and shaken at $4^{\circ} \mathrm{C}$ overnight. Complete and incomplete Freund's adjuvants were purchased from Difco (Difco Laborstories, Detroit, USA), and MPL adjuvant was prepared as described earlier [12]. Control treatments were also prepared as follows: 1. conjugate solution without adjuvant, 2. unconjugated mixture of O-PS and rEPA with MPL adjuvant, and 3. sterile PBS. Six to eight week old female BALB/c mice (ten animals per group) were immunized with the conjugates emulsified with the adjuvants and with the control treatments (200 $\mu 1 /$ mouse/dose, injected i.p. at two sites) on days 0 , 21, and 42. Complete Freund's adjuvant was used in immunization and incomplete Freund's adjuvant was used in booster injections. Blood samples were withdrawn pre-injection and 14 days after each injection, centrifuged at $3000 \mathrm{rpm}$ for 10 minutes, and stored at $-20^{\circ} \mathrm{C}$.

\subsection{Enzyme Linked Immunosorbant Assay (ELISA)}

Specific IgM and IgG sera titers were evaluated with their homologous LPS using ELISA as described earlier [28]. Serum end point titers were calculated as the reciprocal of the serum dilution where the optical density of the assay equaled the average value of the control plus three standard deviations.

The whole bacterial cell ELISA was performed as follows: Freshly grown bacterial cells were killed by $0.3 \%$ formalin at $4^{\circ} \mathrm{C}$ overnight. The cells were centrifuged at $5000 \mathrm{rpm}$ at $4^{\circ} \mathrm{C}$ for $10 \mathrm{~min}$ and washed once with sterile PBS. The pellet was resuspended in $20 \mathrm{ml}$ sterile carbonate buffer ( $\mathrm{pH} 9.6)$ and diluted to $\mathrm{A}_{600}$ of 0.45 , which is approximately equivalent to $6 \times 10^{8} \mathrm{CFU} / \mathrm{ml}$. The diluted bacterial cell suspensions were used as the antigen for the ELISA assay.

\subsection{In Vivo Protection of Mice}

The infecting inocula of $P$. aeruginosa IATS strains 6, 10, 11, and 20 in mice were determined in order to perform the in vivo protection test. The $\mathrm{LD}_{50}$ value was determined as described by Reed and Muench [29].

In vivo protection was performed using BALB/c female mice (5 mice per group, 6 - 8 weeks old). Mice were immunized with the prepared conjugates as described above. Two weeks after the last injection, the mice were bled for the sera titer assay and challenged i.p. with 10 times the determined $\mathrm{LD}_{50}$ as described earlier [30]. The mice were monitored daily for 7 days for mortality. 


\subsection{Statistical Analyses}

The experimental data were analyzed by the software programs Prism 5 (Graph Pad) or Excel (Microsoft) and were expressed as mean \pm SE. A P value of $\leq 0.05$ determined by student's test was considered statistically significant.

\section{Results}

\subsection{Isolation and Characterization of LPS}

Smooth type LPSs of $P$. aeruginosa IATS strains $6,10,11$, and 20 were obtained with a yield of approximately $1 \%-2 \%$ LPS based on dry cell weight. DNA and protein contents were determined to be less than $1 \%$ of the prepared LPS, indicating a high purity. Partially hydrolyzed LPSs of IATS strains 6, 10, 11, and 20 produced water soluble O-PSs with a yield of $37 \%, 31 \%, 16 \%$, and $27 \%$, respectively.

\subsection{Characterization of Conjugates}

The preliminary safety test of rEPA carrier protein showed that a dose of up to $400 \mu \mathrm{g}$ did not result in any signs of illness or weight loss in any of the tested mice for 7 days after injection, demonstrating the safety of rEPA for use as a carrier protein.

The O-PS was coupled to rEPA through an ADH spacer by a carbodiimide-mediated condensation reaction. The prepared conjugate was purified by gel filtration chromatography and eluted with PBS in the void volume as a single peak (Figure 1). The peak was positive for both carbohydrate and protein contents, indicating that the conjugation method was successful. Non-conjugated protein and carbohydrate contents were eluted in later fractions as two overlapping peaks.

The sugar and protein contents of the prepared conjugates were determined using phenol sulfuric acid method for sugars [31] and the Bradford assay for proteins [24]. The molar ratios of protein to saccharide moieties in the conjugates were as follow: 1:6 for O-PS 6 -ADH-rEPA, 1:2.3 for O-PS ${ }_{10}$-ADH-rEPA, 1:2.7 for O-PS 11 -ADHrEPA, 1:14 for O-PS ${ }_{20}$-ADH-rEPA, and 1:5 for O-PS poly-ADH-rEPA.

\subsection{Immunogenicity of the MV}

The immunogenicity of the conjugates was evaluated using female BALB/c mice. Levels of IgM and IgG antibodies elicited against the conjugates emulsified with MPL, Freund's adjuvants, or the conjugates without adjuvant were compared with the unconjugated mixture of rEPA/O-PS emulsified with MPL and sterile PBS as negative control. The elicited antibody titers were measured against homologous pure LPS or homologous whole bacterial cells using ELISA. Conjugates emulsified with MPL adjuvant elicited significantly higher levels of anti-O-PS

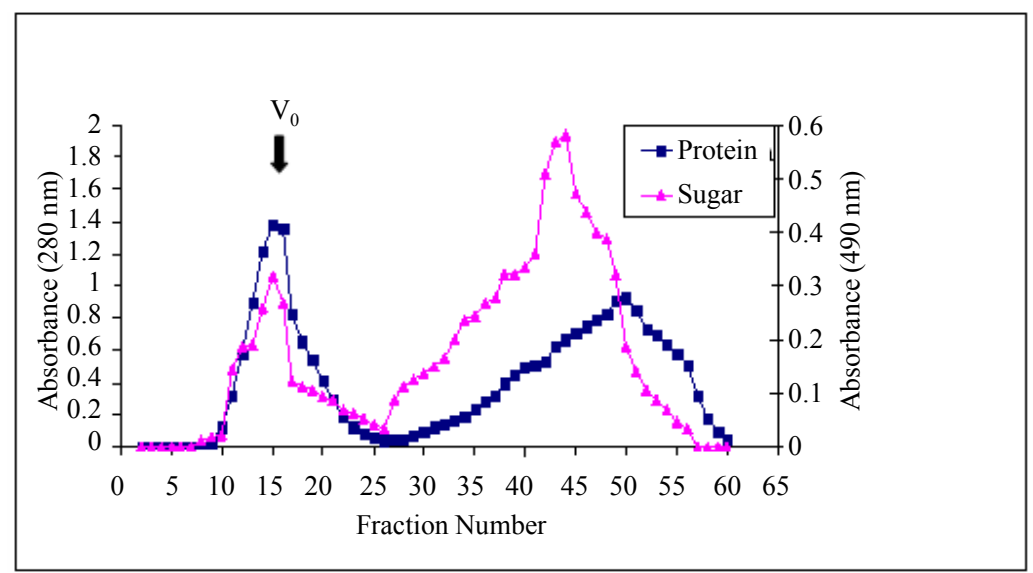

Figure 1. Elution profile of the $\mathrm{O}-\mathrm{PS}_{20}-\mathrm{ADH}-\mathrm{rEPA}$ conjugate derived from O-PS of $P$. aeruginosa IATS strain 20 on Sephadex G-100 eluted with PBS. The protein content was monitored by optical absorbance $\left(\mathrm{A}_{280}\right)$ and sugar content was assayed by the phenol-sulfuric acid method $\left(\mathrm{A}_{490}\right)$. $\mathrm{V}_{\mathrm{O}}$ : void volume. 
IgM titers than others, except in IATS 11, where the non-conjugated treatment elicited the highest titer (Figure 2(a)). As expected, weak anti-O-PS IgM titers were recorded in the PBS negative control.

Similarly, the highest anti-O-PS IgG titers recorded when the conjugates were emulsified with MPL (Figure 2(b)). All conjugate vaccine treatments induced significantly higher levels of anti-LPS IgG titers compared to the unconjugated mixture groups. Analogous to IgM control groups, weak anti-O-PS IgG titers against O-PS were recorded in the PBS negative control groups. Similar results were also obtained using the whole bacterial cell ELISA (Figure 2(c)).

The unconjugated mixture of O-PS/rEPA emulsified with MPL elicited the highest level of anti-rEPA IgG titers, followed by conjugates emulsified with MPL (Figure 3). As expected, the negative control treatments did
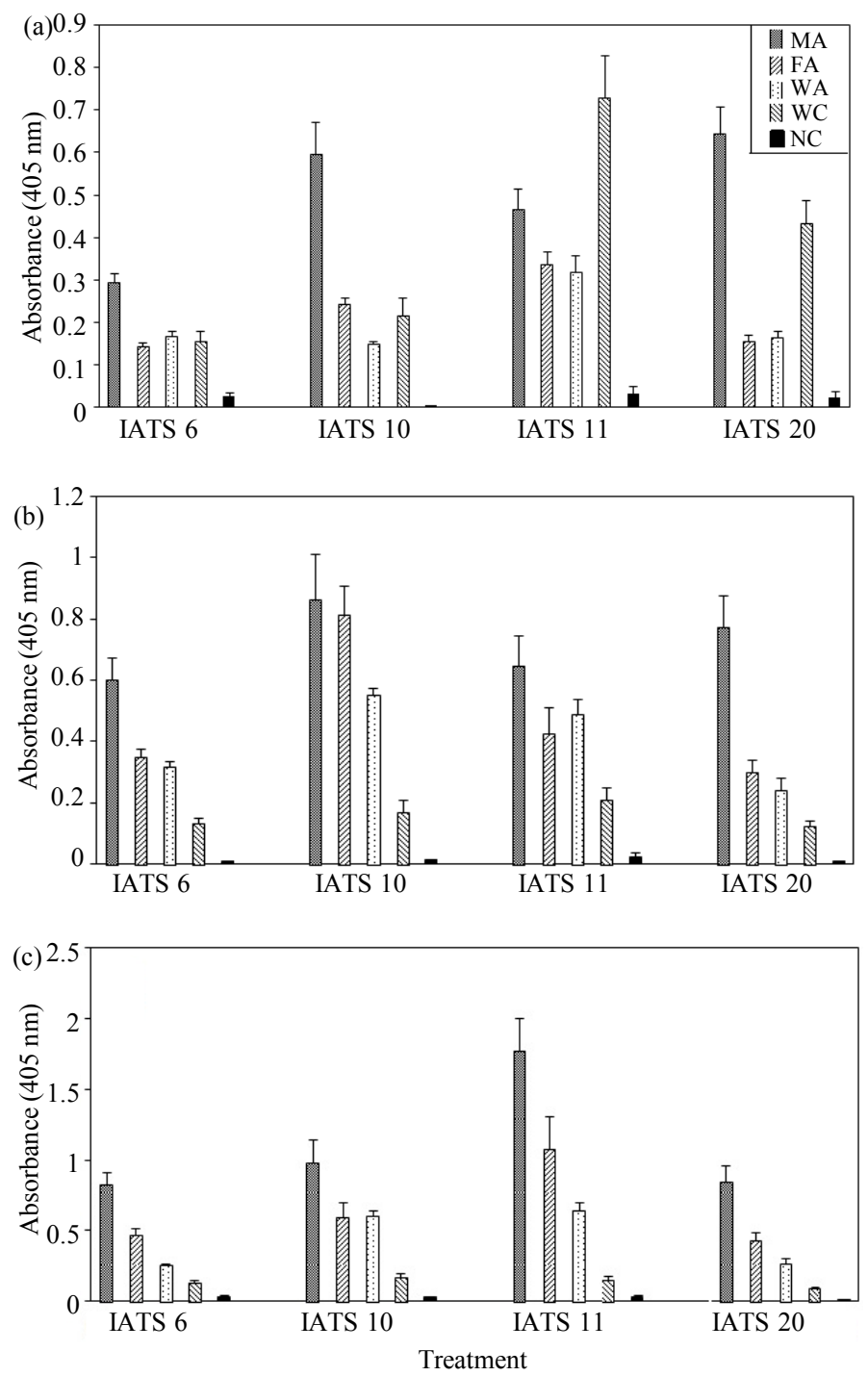

Figure 2. ELISA titers elicited in BALB/c mice against monovalent conjugate vaccines derived from O-PSs of $P$. aeruginosa IATS strains $6,10,11$, and 20. (a) IgM titers measured against homologous pure LPS; (b) IgG titers measured against homologous pure LPS; and (c) IgG titers measured against homologous whole cells. MA: conjugate emulsified with MPL adjuvant; FA: conjugate emulsified with Freund's adjuvants; WA: conjugate without adjuvant; WC: unconjugated mixture of O-PS/rEPA emulsified with MPL, and NC: PBS negative control. The serum dilution is $1 / 1,600$, and the titer level is depicted as $\mathrm{A}_{405}$. Each bar represents the mean \pm standard error for 10 mice sera. 


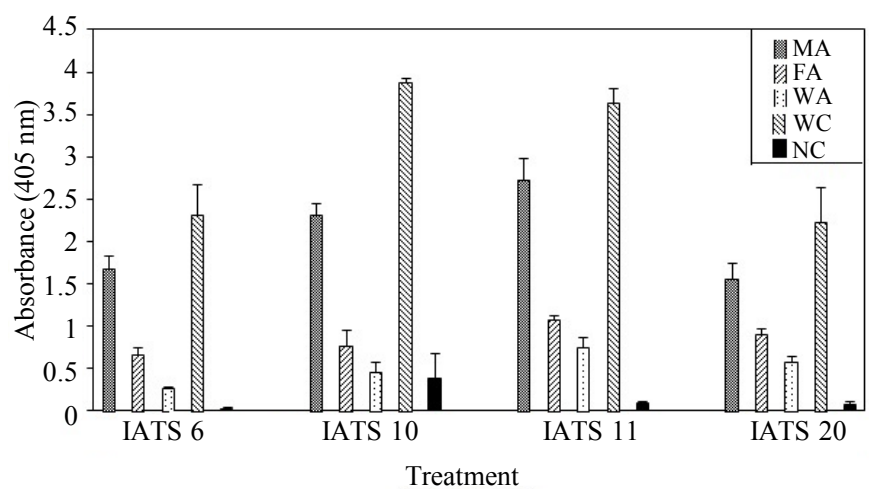

Figure 3. ELISA anti-rEPA IgG titers elicited in BALB/c mice against monovalent conjugate vaccines derived from O-PSs of $P$. aeruginosa IATS strains $6,10,11, \& 20$. The serum dilution is $1 / 25,600$, and the titer level is depicted as $\mathrm{A}_{405}$. Each bar represents the mean \pm standard error for 10 mice sera. Abbreviations are the same as in Figure 2.

not elicit a significant anti-rEPA antibody. The O-PS 10 -ADH-rEPA and O-PS 11 -ADH-rEPA, which showed the lowest O-PS/rEPA molar ratios (see above), elicited the highest level of anti-rEPA antibodies. In contrast, O$\mathrm{PS}_{20}$-ADH-rEPA, which showed the highest O-PS/rEPA molar ratio, elicited the lowest level of anti-rEPA antibodies.

\subsection{Immunogenicity of the PV}

The immunogenicity of the PV was evaluated by immunization of mice with the conjugate emulsified with MPL or Freund's adjuvants, compared with sterile PBS as a negative control. The elicited antibody titers were measured against LPSs or whole cells of $P$. aeruginosa IATS strains 6, 10, 11, and 20 using the ELISA (Figure 4). The PV emulsified with MPL adjuvant elicited a higher level of anti-O-PS IgM than that emulsified with Freund's adjuvants when measured against the LPSs of the four IATS strains as coating antigens (Figure 4(a)). Similarly, the elicited anti-O-PS IgG titers using MPL adjuvant were higher than that using Freund's adjuvants in both LPS or whole cell ELISA (Figure 4(b) and Figure 4(c)), with the exception of the case when LPS of IATS 20 was used as a coating antigen, in which both sera showed very close titer levels. As expected, very weak anti-O-PS IgM and IgG titers were observed in the PBS negative control group.

The data on the immunogenicity of the MV and PV conjugates indicate that MPL adjuvant is the most efficient to elicit IgM and IgG against O-PS moieties in the conjugates, followed by Freund's adjuvants.

\subsection{In Vivo Protection of Mice}

The $\mathrm{LD}_{50}$ for $P$. aeruginosa was determined according to Reed and Muench [29] method. The data were derived from two separate experiments. The $\mathrm{LD}_{50}$ of $P$. aeruginosa IATS strains $6,10,11$, and 20 were found to be $3.6 \times$ $10^{7}, 2.3 \times 10^{7}, 1.56 \times 10^{8}$, and $3.7 \times 10^{7} \mathrm{CFU} /$ mouse, respectively. The differences in the $\mathrm{LD}_{50}$ values between all strains used in this study were less than one $\log$. Ten times the determined $\mathrm{LD}_{50}$ values were used as doses for the in vivo protection studies.

Immunization of mice with the prepared MV derived from O-PSs of IATS strains 10, 11, and 20 emulsified with MPL or Freund's adjuvants provided a high level of protection against the homologous bacterial strain (Table 1). However, the conjugate of O-PS from IATS 6 provided a lower level of protection against the homologous strain. Lower protective capacities were also obtained when the mice were immunized with conjugates without adjuvant or unconjugated O-PS/rEPA with MPL adjuvant (Table 1), where all non-immunized animals died.

The in vivo cross protection of the conjugates was assayed with the heterologous bacterial strain. The prepared conjugates of IATS strains 6, 10, and 20 emulsified with MPL allowed survival of 2/5, 4/5, and 5/5 of tested animals, respectively, challenged with IATS 11 bacterial strain. The low level of protection provided by the IATS 6 conjugate and the high level of protection provided by the IATS 10 and 20 conjugates against the 

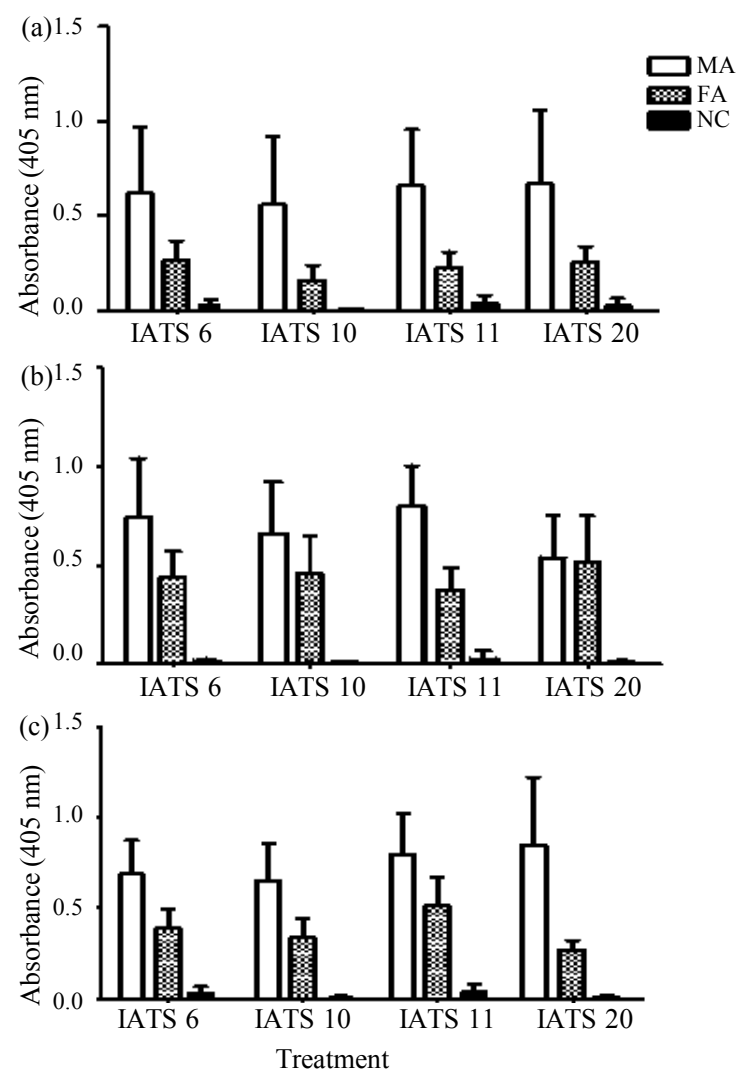

Figure 4. ELISA titers elicited in BALB/c mice against polyvalent conjugate vaccines derived from a mixture of O-PSs from $P$. aeruginosa IATS strains $6,10,11$, and 20 with rEPA protein. (a) IgM titers measured against LPS coating antigen; (b) IgG titers measured against LPS coating antigen; and (c) IgG titers measured against whole cell antigen. The serum dilution is $1 / 1,600$, and the titer level is depicted as $\mathrm{A}_{405}$. Each bar represents the mean \pm standard error for 10 mice sera. Abbreviations are the same as in Figure 2.

Table 1. Protective capacity of monovalent conjugate vaccines against a $P$. aeruginosa homologous strain injected at tenfold its $\mathrm{LD}_{50}$.

\begin{tabular}{|c|c|c|c|c|}
\hline $\begin{array}{l}\text { Protective } \\
\text { Conjugate } \\
\text { Treatment }\end{array}$ & $\begin{array}{c}\mathrm{O}^{\mathrm{P}} \mathrm{PS}_{6}-\mathrm{ADH}-\mathrm{rEPA} \\
\text { (survived/total animals) }\end{array}$ & $\begin{array}{c}\text { O-PS }{ }_{10}-\mathrm{ADH}-\mathrm{rEPA} \\
\text { (survived/total animals) }\end{array}$ & $\begin{array}{c}\mathrm{O}-\mathrm{PS}_{11}-\mathrm{ADH}-\mathrm{rEPA} \\
\text { (survived/total animals) }\end{array}$ & $\begin{array}{c}\mathrm{O}^{\mathrm{O}-\mathrm{PS}_{20}-\mathrm{ADH}-\mathrm{AEPA}} \\
\text { (survived/total animals) }\end{array}$ \\
\hline MPL adjuvant & $3 / 5$ & $5 / 5$ & $5 / 5$ & $5 / 5$ \\
\hline Freund's adjuvant & $2 / 5$ & $5 / 5$ & $3 / 5$ & $4 / 5$ \\
\hline No adjuvant & $0 / 5$ & $3 / 5$ & $2 / 5$ & $3 / 5$ \\
\hline $\begin{array}{l}\text { Unconjugated } \\
\text { O-PS/rEPA }\end{array}$ & $0 / 5$ & $1 / 5$ & $1 / 5$ & $1 / 5$ \\
\hline Negative control & $0 / 5$ & $0 / 5$ & $0 / 5$ & $0 / 5$ \\
\hline
\end{tabular}

heterologous IATS 11 bacterial strain are in agreement with the homologous protective capacity of IATS strains 6, 10, and 20 (Table 1).

Study of in vivo protective capacity of the PV against $P$. aeruginosa IATS strains was also performed. The PV emulsified with MPL allowed survival of $1 / 5,5 / 5,4 / 5$, and $5 / 5$ of tested animals challenged with IATS bacterial strains $6,10,11$, and 20 , respectively, as compared to $0 / 5$ surviving animals in a non-vaccinated group. The low level protection of the PV against the IATS 6 and the high level of protection against IATS strains 10, 11 , and 20 correlate with the homologous and heterologous protective capacities of MV against the bacterial strains, as shown above. 


\section{Discussion}

$P$. aeruginosa is one of the leading opportunistic pathogens in immunocompromised patients. Despite the development of antimicrobial therapy to control bacterial infections, $P$. aeruginosa remains a major problem due to its intrinsic resistance to antibiotics and its ability to acquire resistance to multiple antimicrobial agents [5]. As alternatives to antimicrobial therapy, several methods have been evaluated to protect vulnerable patients from $P$. aeruginosa infections, including passive immunization with antibodies [16], active immunization using various cellular components [2] [18], and preparation of conjugate vaccines [13]-[15]. Conjugate vaccine is recommended for immunization over other immunogens because of its safety and its ability to elicit protective antibodies against the carbohydrate antigen.

LPS of $P$. aeruginosa is immunogenic but highly toxic [6] [9]. Treatment with high molecular weight bacterial PS resulted in some immune response, but not enough to protect from bacterial diseases in either infants or adults [32]. The immunogenicity of PS antigen has been readily enhanced through conjugation of the PS antigen to protein carriers [13] [14]. Many investigators have conjugated O-PS from P. aeruginosa to various protein carriers such as BSA [12] [16], TT [14], and exotoxin A [13] [15]. Exotoxin A has both immunogenic and cytotoxic effects and is produced by most clinical isolates of $P$. aeruginosa. Unmodified exotoxin A was used as a carrier protein in the polyvalent $P$. aeruginosa vaccine candidate [13]. Antibodies against exotoxin A have been shown to provide protection from $P$. aeruginosa infection [17]. In this study, the nontoxic rEPA was used as a carrier protein in O-PS conjugation due to its safety, immunogenicity, and ability to be used in the development of a multi-antigenic conjugate vaccine to provide broad-spectrum protection from toxic exotoxin A proteins of various $P$. aeruginosa strains [19].

Several conjugation approaches were used to couple the O-PS to a carrier protein. One approach is using reductive amination method [13]. Such approach might nonspecifically oxidize some of the sugar components of the O-specific chain, causing nonspecific alteration on the chemical structure of the O-PS antigen. Berna Biotech developed an octvalent $P$. aeruginosa vaccine candidate (Aerugen ${ }^{\circledR}$ ) by coupling O-PSs to exotoxin A using reductive amination method [13] [33]. Further development in Aerugen ${ }^{\circledR}$ vaccine was suspended because the lack of sufficient efficacy in Phase 3 clinical trials [2]. We used significantly different approach in which the O-PS was coupled through the terminal carboxyl group of the KDO to the nontoxic rEPA carrier protein via ADH. Thus, the O-specific chain remains unaltered.

The immunogenicity of the prepared MV and PV conjugates was evaluated in combination with MPL or Freund's adjuvants using a mouse model. The highest IgM and IgG titers against O-PS moieties were obtained when the conjugates were emulsified with MPL adjuvant, in agreement with many previous studies (Figure 2) [12] [34] [35]. A mixture of unconjugated rEPA and O-PS emulsified with MPL adjuvant elicited the highest level of anti-rEPA IgG, followed by conjugates emulsified with MPL (Figure 3). The level of elicited anti-rEPA $\mathrm{IgG}$ is inversely correlated to the ratio of the coupled O-PS to rEPA protein, which might indicate possible structural alteration or blocking of rEPA epitopes by the coupled O-PS. It is evident that all MVs elicited high levels of IgG titers against homologous O-PS and rEPA antigens (Figure 2 and Figure 3), which might provide multiple mechanisms of protection against $P$. aeruginosa infection. In addition, the anti-rEPA IgG provides broad spectrum cross protection against various strains of $P$. aeruginosa due to its common exotoxin A antigen [17] [19].

No significant differences were observed in IgG levels when using formalin-killed bacteria or pure LPS as coating antigens (Figure 2(b) and Figure 2(c)), which provides evidence that O-PS antigen retained its native conformation after the LPS extraction and conjugation procedure.

The level of antibodies in the host blood has been found to correlate with the level of protection against infection [15]. Therefore, the degree of protection of the conjugates was also evaluated by the level of resistance of mice to infection with $P$. aeruginosa. A high level of protection was obtained against the homologous bacterial strain when mice were immunized with conjugates derived from O-PSs of IATS 10, 11, and 20 emulsified with MPL or Freund's adjuvants, whereas a lower degree of protection was obtained using O-PS derived from the IATS 6 serotype. The degree of in vivo protection was found to be in good correlation with the elicited anti-O-PS IgG level in the serum (Figure 2(b)), which is in a good agreement with the previous studies using the O-PSs of $P$. aeruginosa IATS 6 and 11 coupled to BSA [12], fusion protein of exotoxin A and flagellin of $P$. aeruginosa [18], and O-PS-TT conjugates of Vibrio cholerae O139 [36].

In previous studies, no cross protection was observed between LPSs of $P$. aeruginosa serotypes 0 [10] [12] 
[14]. Nevertheless, the prepared conjugates derived from O-PSs of IATS 10 and 20 emulsified with MPL showed high in vivo cross protection against $P$. aeruginosa IATS 11 . The observed cross protections in the present study might be attributed to the level of IgG titer against rEPA, the common antigen of $P$. aeruginosa strains, which is detected in all mice immunized by the prepared conjugates (Figure 3).

PV from various serotypes may have a potential to stimulate the immune response and protect against many serotypes. Since no cross-reaction was observed between LPSs of different $P$. aeruginosa serotypes 0 [10] [12] [14], a PV containing the LPS antigens of the most prevalent pathogenic serotypes might provide a broad spectrum of protection against a wide variety of pathogenic $P$. aeruginosa serotypes. Furthermore, PV created with rEPA as a carrier protein may evoke both anti-O-PS and anti-rEPA antibodies that may provide extra cross protection against various $P$. aeruginosa serotypes. In previous study [38], PV using LPS antigens elicited high levels of anti-PV in rabbits and humans, which provided sufficient protection of patients at high risk of $P$. aerugi-

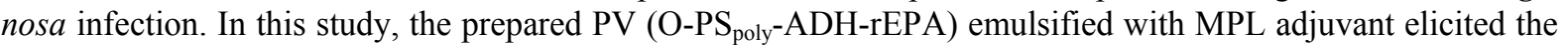
highest anti-O-PS antibodies against LPSs of various $P$. aeruginosa serotypes (Figure 4), which provided a high degree of in vivo protection against living $P$. aeruginosa IATS strains 10,11, and 20, but a low level of protection against IATS strain 6 . The protection capacity of PV is in good correlation with the homologous and heterologous protective capacities of the prepared MVs (see above). The level of protection correlated well with the level of anti-O-PS and anti-rEPA antibodies in the serum of the mice, suggesting that PV may be a good candidate for protection against $P$. aeruginosa infection.

\section{Conclusion}

The immunogenicity and in vivo cross protection of the PV support the use of a multi-antigenic approach for the development of a protective conjugate vaccine. The vaccine elicited high titers of specific antibodies to rEPA and the O-PSs that were able to provide cross protection of mice against various strains of the pathogens. However, it may be necessary to carry out further studies to include other O-PSs from other strains, and to evaluate the degree of cross protection against a wider spectrum of pathogenic strains.

\section{Acknowledgements}

This work was partially supported by Deanship of Academic Research, University of Jordan, Amman, Jordan.

\section{References}

[1] Grimwood, K., Kyd, J.M., Owen, S.J., Massa, H.M. and Cripps, A.W. (2015) Vaccination against Respiratory Pseudomonas aeruginosa Infection. Human Vaccines \& Immunotherapeutics, 11, 14-20. http://dx.doi.org/10.4161/hv.34296

[2] Sharma, A., Krause, A. and Worgall, S. (2011) Recent Developments for Pseudomonas Vaccines. Human Vaccines, 7, 999-1011. http://dx.doi.org/10.4161/hv.7.10.16369

[3] Cryz, S. (1984) Pseudomonas aeruginosa Infections. In: Germanier, R., Eds., Bacterial Vaccine, Academic Press, New York, 317-351. http://dx.doi.org/10.1016/b978-0-12-280880-7.50016-x

[4] Farjah, A., Owlia, P., Siadat, S.D., Mousavi, S.F., Ardestani, M.S. and Mohammadpour, H.K. (2015) Immunological Evaluation of an Alginate-Based Conjugate as a Vaccine Candidate against Pseudomonas aeruginosa. Acta Pathologica, Microbiologica et Immunologica Scandinavica, 123, 175-183. http://dx.doi.org/10.1111/apm.12337

[5] Tenover, F.C. (2006) Mechanisms of Antimicrobial Resistance in Bacteria. American Journal of Infection Control, 34, S3-S10. http://dx.doi.org/10.1016/j.ajic.2006.05.219

[6] Cryz Jr., S.J., Furer, E. and Germanier, R. (1984) Protection against Pseudomonas aeruginosa Burn Wound Sepsis by Immunization with Lipopolysaccharide and High-Molecular-Weight Polysaccharide. Infection and Immunity, 43, 795799.

[7] Campodónico, V.L., Llosa, N.J., Bentancor, L.V., Maira-Litrán, T. and Pier, G.B. (2011) Efficacy of a Conjugate Vaccine Containing Polymannuronic Acid and Flagellin against Experimental Pseudomonas aeruginosa Lung Infection in Mice. Infection and Immunity, 79, 3455-3464. http://dx.doi.org/10.1128/IAI.00157-11

[8] Farjah, A., Owlia, P., Siadat, S.D., Mousavi, S.F. and Shafieeardestani, M. (2014) Conjugation of Alginate to a Synthetic Peptide Containing T- and B-Cell Epitopes as Induction for Protective Immunity against Pseudomonas aeruginosa. Journal of Biotechnololgy, 192, 240-247. http://dx.doi.org/10.1016/j.jbiotec.2014.10.025

[9] Priebe, G.P., Walsh, R.L., Cederroth, T.A., Kamei, A., Coutinho-Sledge, Y.S., Goldberg, J.B. and Abd-Pier, G.B. 
(2008) IL-17 Is a Critical Component of Vaccine-Induced Protection against Lung Infection by LipopolysacchrideHeterologous Strains of Pseudomonas aeruginosa. The Journal of Immunology, 181, 4965-4975. http://dx.doi.org/10.4049/jimmunol.181.7.4965

[10] Fisher, M.W., Devlin, H.B. and Gnabasik, F.J. (1969) New Immunotype Scheme for Pseudomonas aeruginosa Based on Protective Antigens. Journal of Bacteriolology, 98, 835-836.

[11] Knirel, Y. (1990) Polysaccharide Antigens of Pseudomonas aeruginosa. Critical Reviews in Microbiology, 17, $273-$ 304. http://dx.doi.org/10.3109/10408419009105729

[12] Jennings, H.J. (1990) Capsular Polysaccharides as Vaccine Candidates. Current Topics in Microbiology and Immunology, 150, 97-127. http://dx.doi.org/10.1007/978-3-642-74694-9 6

[13] Al-Zeer, M. and Masoud, H. (2007) LPS-Based Conjugate Vaccines Composed of O-Polysaccharide from Pseudomonas aeruginosa IATS 6 and 11 Bound to a Carrier Protein. World Journal of Microbiology and Biotechnology, 23, 1541-1549. http://dx.doi.org/10.1007/s11274-007-9399-2

[14] Cryz Jr., S.J., Furer, E., Sadoff, J. and Germanier, R. (1986) Pseudomonas aeruginosa Immunotype 5 Polysaccharide-Toxin A Conjugate Vaccine. Infection and Immunity, 52, 161-165.

[15] Cryz Jr., S.J., Sadoff, J., Furer, E. and Germanier, R. (1986) Pseudomonas aeruginosa Polysaccharide-Tetanus Toxoid Conjugate Vaccine: Safety and Immunogenicity in Humans. The Journal of Infectious Diseases, 154, 682-688. http://dx.doi.org/10.1093/infdis/154.4.682

[16] Cryz, S., Lang, A., Sadoff, J., Germanier, R. and Furer, E. (1987) Vaccine Potential of Pseudomonas aeruginosa O-PS-Toxin A Conjugate. Infection and Immunity, 55, 1547-1551.

[17] Tsay, G. and Collins, M. (1984) Preparation and Characterization of Non Toxic PS-Protein Conjugate That Induces Active Immunity and Passively Protective Antibody against Pseudomonas aeruginosa Immunotype 1 in Mice. Infection and Immunity, 45, 217-221.

[18] Pollack, M. (1983) The Role of Exotoxin A in Pseudomonas Disease and Immunity. Reviews of Infectious Diseases, 5, S979-S984. http://dx.doi.org/10.1093/clinids/5.supplement 5.s979

[19] Farajnia, S., Peerayeh, S.N., Tanomand, A., Majidi, J., Goudarzi, G., Naghili, B. and Rahbarnia, L. (2015) Protective Efficacy of Recombinant Exotoxin A-Flagellin Fusion Protein against Pseudomonas aeruginosa Infection. Canadian Journal of Microbiology, 61, 60-64. http://dx.doi.org/10.1139/cjm-2014-0501

[20] Isakov, M.A., Soldatenkova, A.S., Kaloshin, A.A. and Mikhailova, N.A. (2011) Immunobiological Properties of Recombinant Atoxicforms of the Pseudomonas aeruginosa Exotoxin A. Zhurnal Mikrobiologii, Epidemiologii, i Immunobiologii, 2, 37-42.

[21] Micoli, S., Rondini, S., Pisoni, I., Proietti, D., Berti, F., Costantino, P., Rappuoli, R., Szu, S., Saul, A. and Martin, L.B. (2011) Vi-CRM 197 as a New Conjugate Vaccine against Salmonella typhi. Vaccine, 29, 712-720. http://dx.doi.org/10.1016/j.vaccine.2010.11.022

[22] Szu, S.C. (2013) Development of Vi Conjugate-A New Generation of Typhoid Vaccine. Expert Review of Vaccines, 12, 1273-1286. http://dx.doi.org/10.1586/14760584.2013.845529

[23] Westphal, O. and Jann, K. (1965) Bacterial Lipopolysaccharides Extraction with Phenol-Water and Further Applications of the Procedure. Methods in Carbohydrate Chemistry, 5, 83-91.

[24] Sambrook, J., Fritsch, E. and Maniatis, T. (1989) Molecular Cloning: A Laboratory Manual. 2nd Edition, Cold Spring Harbor Laboratory Press, New York.

[25] Bradford, M. (1976) A Rapid and Sensitive Method for the Quantitation of Microgram Quantities of Protein Utilizing the Principle of Protein-Dye Binding. Analytical Biochemistry, 72, 248-254. http://dx.doi.org/10.1016/0003-2697(76)90527-3

[26] Gu, X. and Tsai, C. (1993) Preparation, Characterization, and Immunogenicity of Meningococcal Lipooligosaccharide Derived OS-Protein Conjugate. Infection and Immunity, 61, 1873-1880.

[27] Masoud, H., Sadovskaya, T., DeKievit, E., Altman, J., Richards, J. and Lam, J. (1995) Structural Elucidation of the Lipopolysaccharide Core Region of the O-Chain Deficient Mutant Strain A28 from Pseudomonas aeruginosa Serotype O6 (International Antigenic Typing Scheme). Journal of Bacteriolology, 177, 6718-6726.

[28] Dasgupta, T., De Kievit, T.R., Masoud, H., Altman, E., Richards, J.C., Sadovskaya, I., Speert, D.P. and Lam, J.S. (1994) Characterization of Lipopolysaccharide-Deficient Mutants of Pseudomonas aeruginosa Derived from O3, O5, and O6. Infection and Immunity, 62, 809-817.

[29] Plested, J., Gindey, M., Coull, P., Griffith, H., Herbert, M., Bird, G., Richards, J.C. and Moxon, E.R. (2000) Enzyme Linked Immunosorbent Assay (ELISA) for the Detection of Serum Antibodies to the Inner Core Lipopolysaccharide of Neisseria meningitidis Group B. Journal of Immunological Methods, 237, 73-84. http://dx.doi.org/10.1016/S0022-1759(00)00142-3 
[30] Reed, L. and Muench, H. (1938) A Simple Method of Estimating 50\% Endpoints. American Journal of Hygiene, 27, 493-497.

[31] Specht, B.-U., Knapp, B., Muth, G., Broker, M., Hungerer, K.-D., Diehl, K.-D., Massarrat, K., Seemann, A. and Domdey, H. (1995) Protection of Immunocompromised Mice against Lethal Infection with Pseudomonas aeruginosa by Active or Passive Immunization with Recombinant $P$. aeruginosa Outer Membrane Protein $\mathrm{F}$ and Outer Membrane Protein I Fusion Proteins. Infection and Immunity, 63, 1855-1862.

[32] Dubios, M., Gales, K., Hamilton, J., Rebers, P. and Smith, F. (1956) Colorimetric Method for Determination of Sugars and Related Substances. Analytical Chemistry, 28, 350-356. http://dx.doi.org/10.1021/ac60111a017

[33] Baker, C.J. and Edwards, M.S. (2003) Group B Streptococcal Conjugate Vaccines. Archives of Disease in Childhood, 88, 375-378. http://dx.doi.org/10.1136/adc.88.5.375

[34] Zuercher, A.W., Imboden, M.A., Jampen, S., Bosse, D., Ulrich, M., Chtioui, H., Lauterburg, B.H. and Lang, A.B. (2006) Cellular Immunity in Healthy Volunteers Treated with an Octavalent Conjugate Pseudomonas aeruginosa Vaccine. Clinical and Experimental Immunology, 143, 132-138. http://dx.doi.org/10.1111/j.1365-2249.2005.02964.x

[35] Yu, S. and Gu, X.-X. (2005) Synthesis and Characterization of Lipopolysaccharide-Based Conjugate Vaccines for Serotype B Moraxella catarrhalis. Infection and Immunity, 73, 2790-2796. http://dx.doi.org/10.1128/IAI.73.5.2790-2796.2005

[36] Gu, X.-X., Tsai, C.-M., Ueyama, T., Barenkamp, S.J., Robbins, J.B. and Lim, D.J. (1996) Synthesis, Characterization, and Immunologic Properties of Detoxified Lipopolysaccharide from Non-Typeable Haemophilus influenzae Conjugated to Proteins. Infection and Immunity, 64, 4047-4052.

[37] Bountonnier, A., Villeneure, S., Nato, F., Dassy, B. and Fournier, J. (2001) Preparation, Immunogenicity, and Protective Efficacy in a Murine Model of a Conjugate Vaccine Composed of the Polysaccharide Moiety of the LPS of Vibrio cholerae O139 Bound to Tetanus Toxiod. Infection and Immunity, 69, 3488-3493. http://dx.doi.org/10.1128/IAI.69.5.3488-3493.2001

[38] MacIntyre, S., McVeigh, T. and Owen, P. (1985) Immunochemical and Biochemical Analysis of the Polyvalent Pseudomonas aeruginosa Vaccine PV. Infection and Immunity, 51, 675-686.

\section{Abbreviations}

IATS, international antigenic typing scheme; LPS, lipopolysaccharide; O-PS, O-polysaccharide; rEPA, recombinant exotoxin A of $P$. aeruginosa; BSA, Bovine serum albumin; KDO, 2-keto-3-deoxyoctulosonic acid; ADH, adipic acid dihydrazide; MPL, monophosphoryl lipid A; Freund, complete and incomplete Freund adjuvants; ELISA, enzyme linked immunosorbant assay; i.p., intraperitoneal; CFU, colony forming units; TT, tetanus toxoid; MV, monovalent; PV, polyvalent; O-PS 6 -ADH-rEPA, O-PS of P. aeruginosa IATS 6 conjugated through ADH to rEPA (same thing with IATS 10, 11, 20 and poly). 